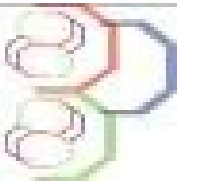

Journal of Applied Biosciences 135: 13821 - 13830

ISSN 1997-5902

\title{
Le système de production de la poule locale contraint son développement au Sud-Kivu, Est de la République Démocratique du Congo
}

\author{
Bisimwa N. Patrick ${ }^{1 *}$, Ayagirwe B. Rodrigue ${ }^{1,2}$, Lugamba R. Trésor ${ }^{1}$, Wasso S. Dieudonné1, Aksanti
} B. Chance 1, Bisimwa B. Espoir1,2

1Université Évangélique en Afrique, Faculté des Sciences Agronomiques et Environnement. B P 3323 Bukavu R D Congo

2Institut Superieur de Développement Rural de Bukavu

Auteur correspondant: Bisimwa N. Patrick ; patrick.ntagereka@ygmail.com

Original submitted in on $21^{\text {st }}$ December 2018. Published online at www.m.elewa.org/journals/ on $31^{\text {st }}$ March 2019 https://dx.doi.org/10.4314/jab.v135i1.8

\section{RÉSUMÉ}

Objectif : Cette étude a été menée envue de caractériser le système de production et de reproduction de la poule locale dans trois zones agro-écologiques du Sud-Kivu d'Avril jusqu'en Décembre, 2017.

Méthodologie et résultats: Une enquête transversale a été menée à l'aide d'une interview auprès de 360 éleveurs répartis dans 3 zones agro-écologiques dont la haute, moyenne et basse altitude. Les principaux résultats trouvés indiquent que ces élevages sont gérés par les hommes, ayant un objectif tourné vers le marché $(90,28 \%)$, comptent 24,38 poules qui sont élevés en divagation $(94,17 \%)$ sans logement spécifique $(81,11 \%)$. Les poules reçoivent une supplémentation alimentaire $(65,28 \%)$ et un complément vitaminique (25,83\%). La pseudopeste aviaire est la maladie la plus fréquente $(89,72 \%)$, suite à l'absence de la vaccination $(96,67 \%)$, les éleveurs recourent à la pharmacopée traditionnelle $(77,5 \%)$. Chaque poule donne 13 œufs par cycle de ponte pour 2,96 cycles par an. 70,37\% des œufs pondus sont incubés pour un taux d'éclosion de $84,50 \%$ et un taux de sevrage de $57,25 \%$, intervenant à 2,52 mois après éclosion. Les épidémies et la prédation sont les principales contraintes auxquelles font face les éleveurs de la poule locale en plus du manque d'appuis par les services étatiques.

Conclusion et application des résultats: Quoique cet élevage soit une activité secondaire et selon les techniques de production traditionnelle avec des faibles performances, il reste primordiale dans l'économie des familles rurales et mérite une attention particulière visant son amélioration. Une stabulation ainsi qu'une alimentation équilibrée permettraient d'accroitre la production et réduire les risques de perte des poules.

Mots clés : Elevage, zones-agro écologiques, Contraintes, poule locale, Sud-Kivu. 
Constraints on the Production system of local chickens in South-Kivu, Eastern of Democratic Republic of Congo

ABSTRACT

Objective: This study was conducted in order to characterize the productive and reproductive system of local chicken in three agro-ecological zones of the South Kivu province from April to December 2017.

Methodology and results: A cross-sectional survey was carried out by interviewing 360 chicken breeders from three agro-ecological zones mainly in the high, middle and lower altitude. The main results showed that this farming sector is led by men with a market-oriented goal $(90.28 \%)$, having an average of 24.38 chickens reared in free range system (94.17\%) without specific housing $(81.11 \%)$. Chickens are supplemented with food (65.28\%) and vitamin supplement (25.83\%). The New castle is the most common disease (89.72\%) affecting this sector due to absence of vaccination $(96.67 \%)$ and traditional pharmacopoeia (77.5\%) is the most used by breeders for the treatments of most of diseases. Furthermore, each chicken gives 13 eggs per spawning cycle for 2.96 cycles per year by which $70.37 \%$ of the eggs laid are incubated for a hatching rate of $84.50 \%$ and a weaning rate of $57.25 \%$, occurring 2.52 months after hatching. Epidemics as well as predation are the main constraints hampering the local chicken production in addition to the lack of support by local government services.

Conclusion and application of findings: Although this production system is secondary and based on the traditional production system, with less performances, it remains key in the economy of rural families and needs particular attention for its improvement. An adequate housing and a balanced diet would increase production and reduce the risk of loss of local chickens in the study region.

Key words: Breeding, agro-ecological zones, constraints, local chicken, Sud-Kivu

\section{INTRODUCTION}

L'aviculture familiale joue un rôle important dans l'économie tant rurale qu'urbaine, elle est exploitée par plus de $80 \%$ des populations en Afrique pour la plupart rurales (Fotsa, 2008), et représente un emploi durant la période de soudure (Guey, 2005). Elle contribue significativement à la sécurité alimentaire, la lutte contre la pauvreté et à la gestion écologique des ressources naturelles (Ngou Ngoupayou, 1990 ; Barua and Yoshimura, 1997; Gueye, 2003). Elle est aussi un moyen d'épargne, d'investissement et d'assurance pour les petits fermiers (Moula, 2012). Les ressources génétiques locales sont principalement représentées par la poule locale domestique (Gallus gallus domesticus) qui pourtant enregistre de faibles performances zootechniques par rapport à celles des races exotiques (Youssao et al., 2009 ; Youssao et al., 2009; Osei-Amponsah et al., 2010). Les races indigènes qui représentent plus de $70 \%$ de la population de volailles mondiales (Ngou Ngoupayou et al., 1990; Tchoumboue et al., 2000) restent malheureusement peu décrites dans la littérature et figurent parmi les ressources génétiques animales les plus menacées du monde et avec risque de perdre des caractères ignorés aujourd'hui et potentiellement utile demain (Fotsa, 2008; Moula et al., 2009; Moula, 2012). Selon la FAO, environ $40 \%$ des races aviaires ont un statut de risque inconnu (FAO, 2010). Bien que les races anciennes de poule soient encore liées aux valeurs culturelles, aux origines géographiques et adaptées à l'environnement local, beaucoup d'entre-elles ont subi une diminution importante de la taille de leurs populations et par conséquent, une érosion de leur diversité génétique (Larivière et Leroy, 2008). Au Maghreb comme dans le reste du monde, l'élevage de la volaille est particulièrement frappé par cette érosion génétique, en raison de la généralisation de l'utilisation des souches hybrides et exotique (Bessadok et al., 2003). En Belgique, où cette problématique a déjà fait l'objet d'un recensement et d'une quantification, $95 \%$ des races locales de volaille se sont révélées être en danger d'extinction (Lariviere and Leroy, 2005). En Afrique, où le problème n'est pourtant pas moins aigu, la faible priorité politique accordée à l'aviculture traditionnelle fait que ce type de 
recensement est actuellement indisponible (Munyuli et al., 2002 ; Gueye, 2005). Au Sud-Kivu, une diminution de plus de $19 \%$ des effectifs ont été observé entre 2000 et 2010 . Mais cette diminution a été plus importante $(82,8 \%)$ entre 2009 et 2012 (INS, 2015). Les causes de cette diminution des effectifs sont jusque-là non élucidées. Plusieurs auteurs rapportent l'influence de l'environnement, les caractéristiques socio-économiques des élevages, et les performances de reproduction

\section{METHODOLOGIE}

Milieu d'étude: Une enquête transversale a été réalisée dans la province du Sud-Kivu située à l'Est de la RD Congo auprès de 360 éleveurs des poules locales répartis dans trois différentes zones écologiques particulièrement dans le territoire de Kalehe, Uvira et Walunngu (Figure 1). Cette province s'étant sur une superficie de $69.130 \mathrm{Km}^{2}$ et une population de plus de 6432984 habitants, soit une densité moyenne de 93 habitants par $\mathrm{Km}^{2}$. Elle est situé à l'est du pays entre 1036' de latitude Sud et $5^{\circ}$ de latitude Sud d'une part et $26^{\circ} 47^{\prime}$ de longitude Est et $29^{\circ} 20^{\prime}$ de longitude Est d'autre part. La température comme des facteurs probables (Bachir et al., 2011). Ce pendant cela concerne les autres pays où la poule locale a déjà reçu cette importance. Au Sud-Kivu par, leur identification ainsi que la quantification de leurs effets reste une urgence pour toute relance de l'aviculture familiale. Ce travail vise ainsi à caractériser les systèmes de production de la poule locale et son système de reproduction en vue d'identifier les causes de la diminution des effectifs.

moyenne annuelle est de $19^{\circ} \mathrm{C}$, et l'altitude varie entre $773 \mathrm{~m}$ et $3000 \mathrm{~m}$. On y trouve deux saisons à savoir, la saison sèche qui dure 3 mois, de juin à septembre, et la saison des pluies qui dure 9 mois (DSRP, 2005). Quatre différentes localités ont été visitées pour chaque territoire et dans lesquelles 30 éleveurs des poules ont été sélectionnées aléatoirement. Les facteurs clés pour la sélection de ces localités étaient basés sur leur accessibilité, historique en élevage des poules locales ainsi que la densité de la population des poules locales (>20 têtes $/ \mathrm{km}^{2}$ ).

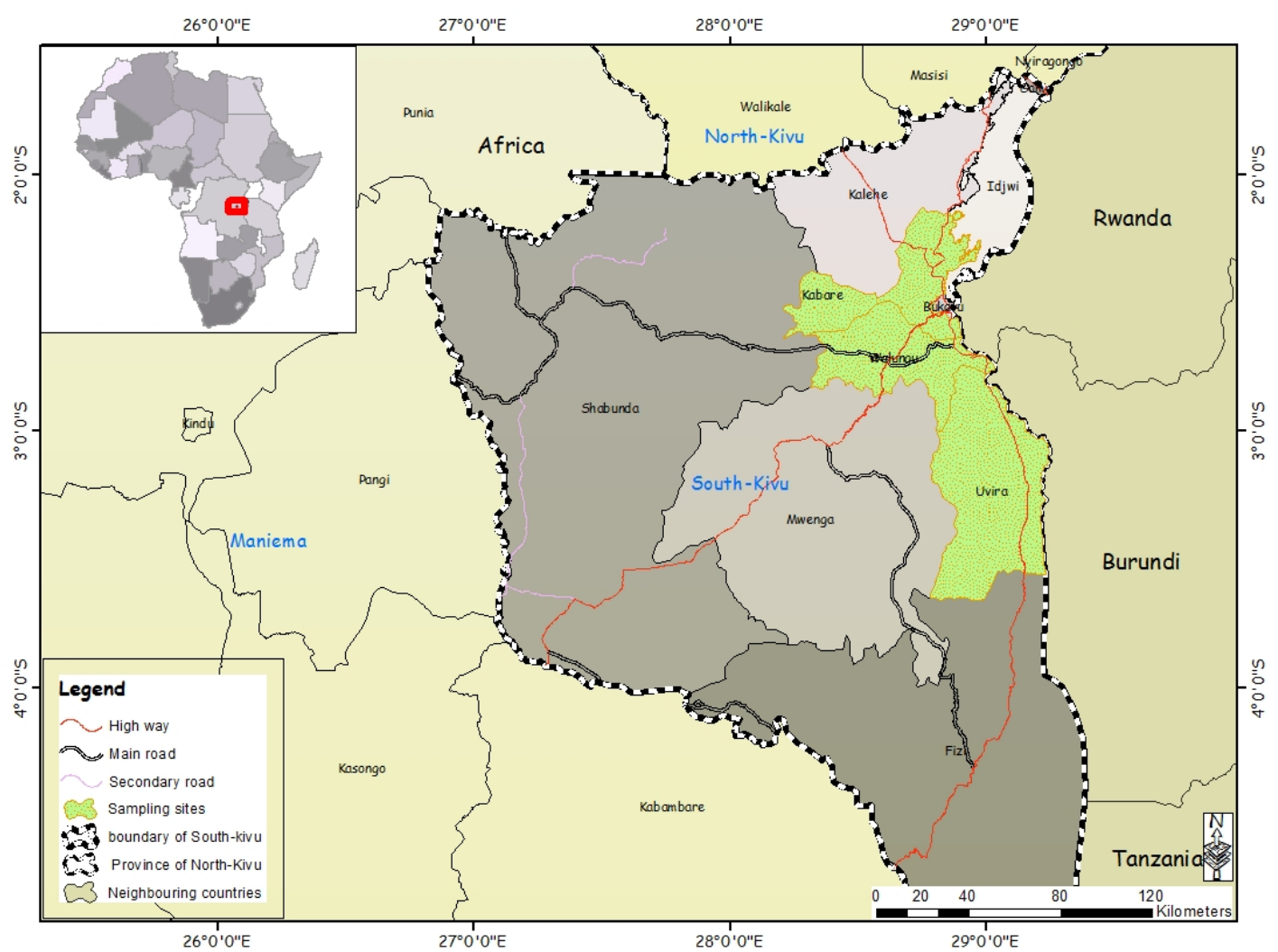

Figure 1: La carte de la province du Sud-Kivu montrant les zones d'étude 

développement au Sud-Kivu, Est de la République Démocratique du Congo

Echantillonnage: La récolte des données a été effectuée à partir du mois d'Avril jusqu'au début du mois de Décembre 2017 dans trois zones agroécologiques. Dans chaque zone, 120 éleveurs ont été choisis aléatoirement selon qu'ils étaient disposés à participer à l'étude. Les interviews ont été réalisées sur base d'un questionnaire traduit oralement en langue locale en cas de besoin. Les principales informations récoltées concernaient les informations sur l'éleveur, le système de production et les caractéristiques de la reproduction. Quelques races locales des poules en élevage traditionnel au Sud-Kivu

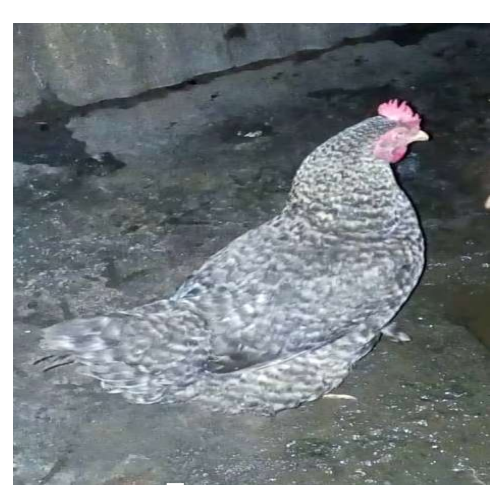

Poulet barré

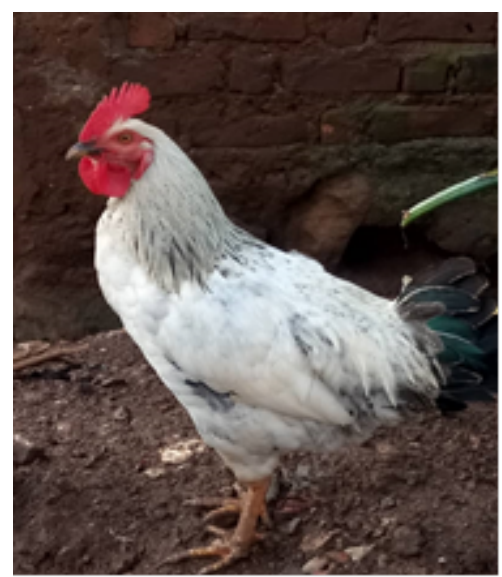

Poulet bourré normale male

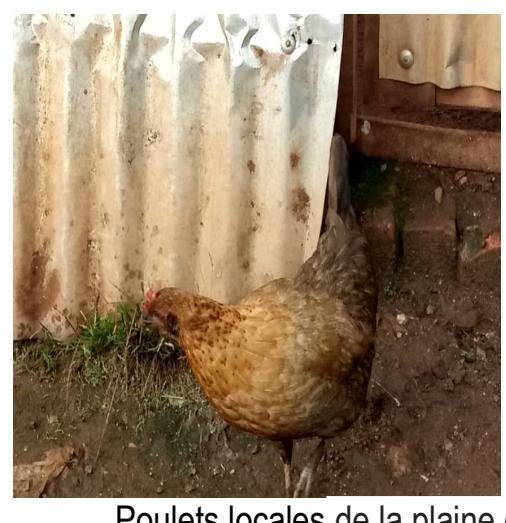

Poulets locales de la plaine de la Ruzizi
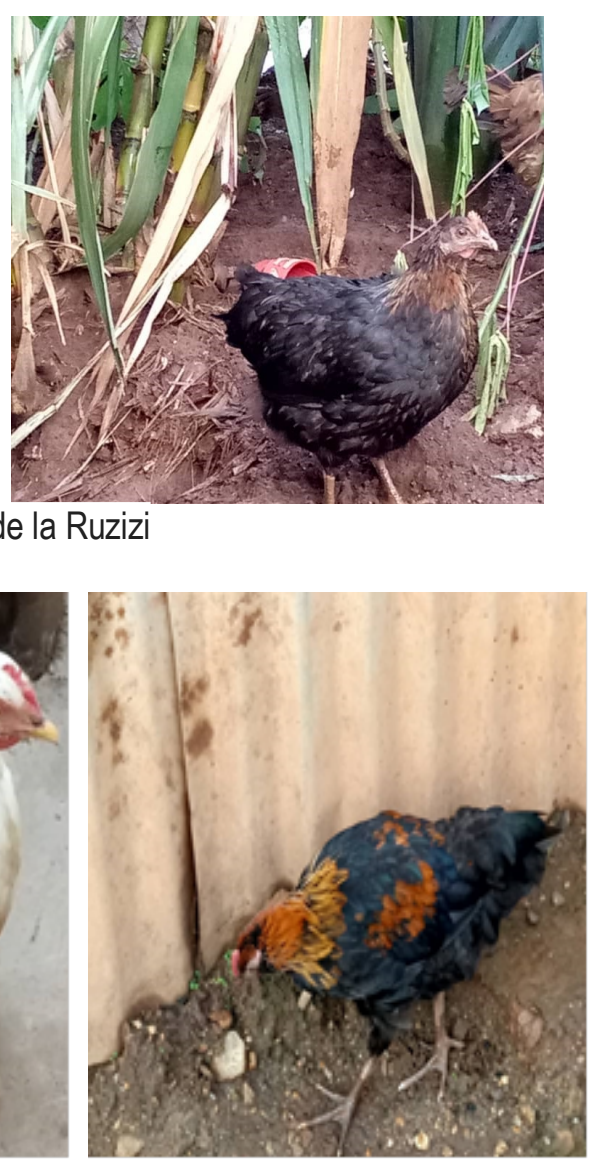

Poulet Ardennais

Poulet Ardennais

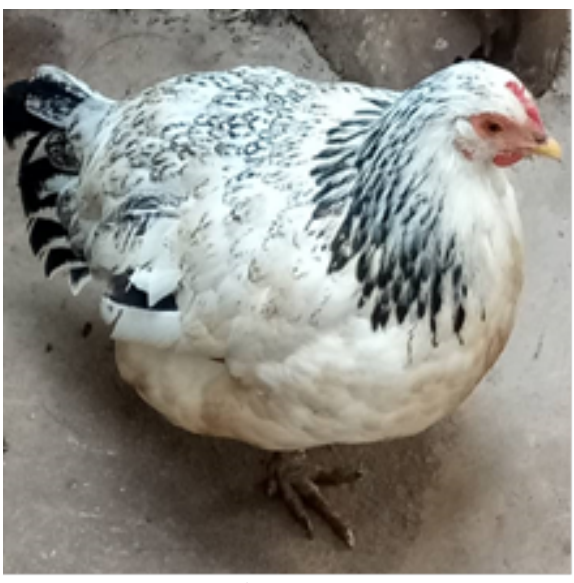

Bourré normale femelle
Analyses statistiques des données: Les données récoltées ont été présentés sous forme de pourcentage par modalité pour les paramètres qualitatifs et sous

\section{RESULATS}

Caractérisation socio-économique des élevages de la poule locale au Sud-Kivu : Au regard des résultats ci-dessus, il ressort que l'élevage des poules locales est détenu principalement par les hommes $(66,6 \%)$ qui sont des mariés $(91,94 \%)$, ayant en moyenne 10,60 ans d'expérience en élevage de poule. Toutefois, la proportion de femmes $(33,33 \%)$ indique que cette activité est inclusive et pourrait contribuer à une intégration du genre. II est une activité secondaire dans la vie de l'exploitant $(65,56 \%)$ de l'agriculture, et est forme de moyenne plus ou moins écartype pour les paramètres quantitatifs. Ces analyses descriptives ont été réalisées en utilisant le logiciel XLSTAT.

pratiqué pour un but commercial $(90,28 \%)$ pour répondre aux autres besoins du ménage, tel que la scolarisation des enfants $(65,00 \%)$, la santé et le soutien aux travaux champêtres $(64,07 \%)$. Chaque ménage élève en moyenne 24,38 poules, avec des variations selon les zones agro-écologiques. Le troupeau est plus dominé par des poules adultes en reproduction et les poussins, alors que les coqs sont les moins représentés car le plus souvent ils sont les plus reformés pour les besoins familiaux. 
Tableau 1. Quelques traits caractéristiques des éleveurs enquêtés

\begin{tabular}{|c|c|c|c|c|c|}
\hline \multirow[t]{2}{*}{ Caractère } & \multirow[t]{2}{*}{ Modalité } & \multicolumn{4}{|c|}{ Zone d'étude } \\
\hline & & Basse altitude & Haute altitude & Moyenne altitude & Moyenne \\
\hline Sexe $(\%)$ & $\begin{array}{l}F \\
M\end{array}$ & $\begin{array}{l}48,33 \\
51,67\end{array}$ & $\begin{array}{l}20 \\
80\end{array}$ & $\begin{array}{l}31,67 \\
68,33\end{array}$ & $\begin{array}{l}33,33 \pm 14,24 \\
66,67 \pm 14,24\end{array}$ \\
\hline Etat-civil (\%) & $\begin{array}{l}\text { Célibataire } \\
\text { Marié } \\
\text { Autres }\end{array}$ & $\begin{array}{l}3,33 \\
93,33 \\
3,33\end{array}$ & $\begin{array}{l}7,5 \\
88,33 \\
4,17\end{array}$ & $\begin{array}{l}5 \\
94,17 \\
0,83\end{array}$ & $\begin{array}{l}5,28 \pm 2,10 \\
91,94 \pm 3,16 \\
2,78 \pm 1,74\end{array}$ \\
\hline Expérience par an & & $9,43 \pm 9,07$ & $15,58 \pm 13,04$ & $6,78 \pm 6,35$ & $10,60 \pm 10,52$ \\
\hline Objectif de l'élevage (\%) & $\begin{array}{l}\text { Vente } \\
\text { Fiente } \\
\text { Don } \\
\text { Consommation }\end{array}$ & $\begin{array}{l}96,67 \\
0 \\
19,17 \\
7,5\end{array}$ & $\begin{array}{l}85,83 \\
7,5 \\
45 \\
51,67\end{array}$ & $\begin{array}{l}88,33 \\
0,83 \\
20 \\
48,33\end{array}$ & $\begin{array}{l}90,28 \pm 5,68 \\
2,78 \pm 4,11 \\
28,06 \pm 14,68 \\
35,83 \pm 24,59\end{array}$ \\
\hline Profession (\%) & $\begin{array}{l}\text { Agriculture } \\
\text { Fonctionnaire } \\
\text { Autres }\end{array}$ & $\begin{array}{l}72,5 \\
13,33 \\
14,17\end{array}$ & $\begin{array}{l}51,67 \\
18,33 \\
30\end{array}$ & $\begin{array}{l}72,5 \\
10 \\
17,5\end{array}$ & $\begin{array}{l}65,56 \pm 12,03 \\
13,89 \pm 4,19 \\
20,56 \pm 8,35\end{array}$ \\
\hline Taille du cheptel & & $28,97 \pm 16,83$ & $23,64 \pm 16,6$ & $20,53 \pm 13,06$ & $24,38 \pm 15,94$ \\
\hline
\end{tabular}

Caractéristiques des systèmes de production de la poule locale au Sud-Kivu: Les différentes caractéristiques de logement des animaux, leur alimentation ainsi que les caractéristiques de la gestion de leur santé sont reprises au tableau 2.

Tableau 2 : Systèmes de production de la poule locale au Sud-Kivu (\%)

\begin{tabular}{l|l|l|l|l|l}
\hline Caractères & Modalité & \multicolumn{2}{l}{ Zone d'étude } & \multicolumn{2}{l}{} \\
\cline { 3 - 6 } & & $\begin{array}{l}\text { Basse } \\
\text { altitude }\end{array}$ & $\begin{array}{l}\text { Haute } \\
\text { altitude }\end{array}$ & $\begin{array}{l}\text { Moyenne } \\
\text { altitude }\end{array}$ & Moyenne \\
\hline Système d'élevage & Stabulation & 0,83 & 3,33 & 1,67 & $1,94 \pm 1,27$ \\
& Divagation & 97,5 & 93,33 & 91,67 & $94,17 \pm 3,00$ \\
& Semi-stabulation & 1,67 & 3,33 & 6,67 & $3,89 \pm 2,55$ \\
\hline Logement ou poulailler & Oui & 20,83 & 23,33 & 12,5 & $18,89 \pm 5,67$ \\
& Non & 79,17 & 76,67 & 87,5 & $81,11 \pm 5,67$ \\
\hline Supplément & Oui & 55,83 & 80 & 60 & $65,28 \pm 12,92$ \\
& Non & 44,17 & 20 & 40 & $34,72 \pm 12,92$ \\
\hline Complément & Vitamines & 9,16 & 55,84 & 12,5 & $25,83 \pm 26,04$ \\
& Aucun & 90,84 & 44,16 & 87,5 & $74,16 \pm 26,04$ \\
\hline Mode d'alimentation & Matin-soir & 58,36 & 48,75 & 57,37 & $54,83 \pm 5,29$ \\
& Matin & 24,99 & 43,74 & 19,66 & $29,47 \pm 12,65$ \\
& Soir & 16,64 & 7,49 & 22,95 & $15,70 \pm 7,77$ \\
\hline Maladie fréquente & Pseudopeste aviaire & 88,33 & 90 & 90,83 & $89,72 \pm 1,27$ \\
& Goumboro & 0 & 0 & 0,83 & $0,28 \pm 0,48$ \\
& Coccidiose & 0,83 & 2,5 & 0,83 & $1,39 \pm 0,96$ \\
& Autres & 17,5 & 12,5 & 10 & $13,33 \pm 3,82$ \\
\hline Période critique & Saison sèche & 47,5 & 27,5 & 62,5 & $45,83 \pm 17,56$ \\
& Saison pluvieuse & 0,83 & 51,67 & 9,17 & $20,56 \pm 27,27$ \\
& toute l'année & 51,67 & 20,83 & 25 & $32,5 \pm 16,73$ \\
\hline
\end{tabular}


Bisimwa et al., J. Appl. Biosci. 2019 Le système de production de la poule locale contraigne son développement au Sud-Kivu, Est de la République Démocratique du Congo

\begin{tabular}{l|l|l|l|l|l}
\hline Palliative en cas de & Vente & 49,17 & 29,17 & 30 & $36,21 \pm 11,32$ \\
maladie & Abattage & 10 & 10,83 & 5,83 & $8,89 \pm 2,68$ \\
& Prévention & 12,5 & 45,83 & 40,83 & $33,06 \pm 17,97$ \\
& Aucune action & 33,33 & 17,5 & 37,5 & $29,53 \pm 10,55$ \\
\hline Traitement & Chimiothérapie & 12,5 & 31,67 & 23,33 & $22,5 \pm 9,61$ \\
& Phytothérapie & 87,5 & 68,33 & 76,67 & $77,5 \pm 9,61$ \\
\hline Utilisation vaccin & Oui & 2,5 & 4,17 & 3,33 & $3,33 \pm 0,84$ \\
& Non & 97,5 & 95,83 & 96,67 & $96,67 \pm 0,84$ \\
\hline Service vétérinaire & Présent & 3,33 & 5 & 6,67 & $5 \pm 1,67$ \\
& Absent & 96,67 & 95 & 93,33 & $95 \pm 1,67$ \\
\hline
\end{tabular}

Les résultats du tableau 2 indiquent que dans les trois zones agro écologiques, la divagation $(94,17 \%)$ est le système de l'élevage le plus utilisé en aviculture familiale. Les poules sont abandonnés à leur sort le matin et ne bénéficient guère d'un habitat, la majorité des éleveurs $(81,11 \%)$ utilisent leur propre maison d'habitation ou la cuisine pour abriter les poules, alors que d'autres passent la nuit sous les arbres. Seulement un nombre réduit d'éleveurs $(18,89 \%)$ construisent des petites maisonnettes pour épargner leurs poules du vol et prédations. En général, $32,04 \%$ des éleveurs ne donnent rien à leurs poules alors que $65,28 \%$ supplémentent l'alimentation de leurs poules surtout en période de récolte où les aliments sont disponibles localement. Les aliments les plus distribués, généralement le matin lorsque les poules sortent de la maison et le soir $(54,83 \%)$ sont entre autre le maïs, les tubercules de manioc, les résidus de cuisine et le riz. A part ces aliments, 25,83\% des éleveurs complémentent aussi leurs poules avec des compléments vitaminiques. La santé dans les élevages reste très fragile. Elle est dominée par la présence de la peste aviaire $(89,72 \%$ des élevages), dont les conséquences sont généralement la perte de tout le cheptel, décourageant ainsi la plupart des éleveurs dans la continuité de cette activité. Observé durant toute l'année, les épidémies les plus importantes et qui occasionnent plus de perte sont observées en fin de la saison sèche $(45,83 \pm 17,56 \%)$. Lorsque l'épidémie est déjà déclarée, certains éleveurs $(36,21 \pm 11,32 \%)$ souhaitent vendre à faible prix leurs poules au marché pour éviter plus de pertes, $33,06 \%$ recourent à la pharmacopée traditionnelle alors que d'autres par contre abattent leur animaux pour la consommation du ménage. Plus de $77,5 \%$ des éleveurs font recours à la pharmacopée traditionnelle (pour des tentatives de traitement) qui est beaucoup plus rependue et accessible à la population et seulement $22,5 \%$ font recours à la chimiothérapie moderne. Seulement 3,33\% font vacciner leurs poules alors que les services vétérinaires ne sont accessibles que pour seulement $5 \%$ de la population en plus il y a absence d'encadrement technique des exploitants en terme de formation sur la conduite de l'élevage

Performance de reproduction de la poule locale au Sud-Kivu: Le tableau 3 révèle que $76,39 \%$ des éleveurs ne font la sélection des reproducteurs, ce qui explique une grande variabilité au sein de la population. En moyenne une poule pond 9,67 à 16,77 œufs par cycle de ponte pour une moyenne de 2,97 cycles par an, soit un total de 41,92 à 50, 08 œufs par an, pour un taux de ponte de 0,86 œuf par jour et par poule mais avec des grandes variabilités selon les zones agroécologiques. Les œufs ne reçoivent pas généralement de protection dans le nid $(54,44 \%)$, quoique certains éleveurs recourent aux couvercles, au retrait des œufs dans le nid, l'utilisation de la braise dans le nid alors que d'autres claustrent le nid. Environ 70,37\% des œufs pondus sont incubés, le choix des œufs à incuber réalisé par seulement $20,56 \%$ des éleveurs porte sur la fraicheur de l'œuf (les derniers pondus sont plus préférés pour l'incubation), le poids, la forme et la grosseur de l'œuf, mais d'autres préfèrent plus utiliser les œufs des races pondeuses exotiques à la place des $œ u f s$ de la poule locale. Les œufs non incubés sont utilisés pour la majorité des cas pour la consommation familiale et la vente. Le taux d'éclosion est de $84,50 \%$, $9,17 \%$ enregistre des casses durant l'incubation mais avec faible une fréquence $(1,30 \%)$ résultant soit d'un mauvais nid, mauvais retournement des œufs par la poule ou alors par écrasement. Si les œufs non éclos sont généralement jetés à la poubelle, ils sont aussi parfois utilisés pour l'alimentation du ménage chez $37,5 \%$ des éleveurs. Le taux de sevrage est de $57,25 \%$ des poussins éclos alors que la mortalité avant sevrage est de $42,57 \%$, la durée de sevrage étant de 2,52 mois. 
Bisimwa et al., J. Appl. Biosci. 2019 Le système de production de la poule locale contraigne son développement au Sud-Kivu, Est de la République Démocratique du Congo

Tableau 3 : Performances reproductives de la poule locale au Sud-Kivu

\begin{tabular}{|c|c|c|c|c|c|}
\hline \multirow[t]{2}{*}{ Caractère } & \multirow[t]{2}{*}{ Modalité } & \multicolumn{4}{|c|}{ Zones d'étude } \\
\hline & & $\begin{array}{l}\text { Basse } \\
\text { altitude }\end{array}$ & Haute altitude & $\begin{array}{l}\text { Moyenne } \\
\text { altitude }\end{array}$ & Moyenne \\
\hline Sélection reproducteur (\%) & $\begin{array}{l}\text { Oui } \\
\text { Non }\end{array}$ & $\begin{array}{l}16,67 \\
83,33\end{array}$ & $\begin{array}{l}40,83 \\
59,17\end{array}$ & $\begin{array}{l}13,33 \\
86,67\end{array}$ & $\begin{array}{l}23,61 \pm 15,01 \\
76,39 \pm 15,01\end{array}$ \\
\hline \multicolumn{2}{|l|}{$\begin{array}{l}\text { OEuf pondu/poule/cycle } \\
\text { Nombre de cycle par an }\end{array}$} & $\begin{array}{l}11,86 \pm 2,95 \\
3,45 \pm 2,95 \\
\end{array}$ & \begin{tabular}{|l|}
$13,63 \pm 3,57$ \\
$2,48 \pm 1,32$ \\
\end{tabular} & $\begin{array}{l}14,16 \pm 3,70 \\
2,97 \pm 1,55 \\
\end{array}$ & $\begin{array}{l}13,22 \pm 3,55 \\
2,96 \pm 1,94\end{array}$ \\
\hline \multicolumn{2}{|l|}{ Fréquence de ponte/jr } & $0,83 \pm 0,24$ & $0,85 \pm 0,23$ & $0,90 \pm 0,20$ & $0,86 \pm 0,22$ \\
\hline Protection des œufs (\%) & $\begin{array}{l}\text { Couvercle } \\
\text { Retrait des œufs } \\
\text { Claustration du nid } \\
\text { Usage de la braise } \\
\text { Aucune }\end{array}$ & $\begin{array}{l}23,33 \\
5 \\
0 \\
0 \\
71,67 \\
\end{array}$ & \begin{tabular}{|l|}
10 \\
15 \\
1,67 \\
51,67 \\
15,83 \\
\end{tabular} & $\begin{array}{l}5 \\
13,33 \\
1,67 \\
4,17 \\
75,83 \\
\end{array}$ & $\begin{array}{l}12,78 \pm 9,48 \\
11,11 \pm 5,36 \\
1,11 \pm 0,96 \\
18,61 \pm 28,70 \\
54,44 \pm 33,50 \\
\end{array}$ \\
\hline \multicolumn{2}{|l|}{ Taux d'incubation (\%) } & $70,60 \pm 15,45$ & $70,15 \pm 15,36$ & $70,48 \pm 14,42$ & $70,37 \pm 15,33$ \\
\hline $\begin{array}{l}\text { Choix des œufs à incuber } \\
(\%)\end{array}$ & $\begin{array}{l}\text { Oui } \\
\text { Non }\end{array}$ & $\begin{array}{l}12,5 \\
87,5\end{array}$ & $\begin{array}{l}22,5 \\
77,5\end{array}$ & $\begin{array}{l}26,67 \\
73,33\end{array}$ & $\begin{array}{l}20,56 \pm 7,28 \\
79,44 \pm 7,28\end{array}$ \\
\hline $\begin{array}{l}\text { Critères de choix des œufs } \\
\text { à incuber }\end{array}$ & $\begin{array}{l}\text { Dernier pondu } \\
\text { Forme et grosseur } \\
\text { OEufs de race } \\
\text { pondeuse }\end{array}$ & $\begin{array}{l}33,36 \\
66,64 \\
0\end{array}$ & $\begin{array}{l}77,78 \\
22,22 \\
0\end{array}$ & $\begin{array}{l}56,24 \\
40,61 \\
3,11\end{array}$ & $\begin{array}{l}59,44 \pm 22,21 \\
39,2 \pm 22,32 \\
1,36 \pm 1,80\end{array}$ \\
\hline \multicolumn{2}{|l|}{ Taux d'éclos (\%) } & $84,86 \pm 16,55$ & $84,78 \pm 14,97$ & $83,86 \pm 15,18$ & $84,50 \pm 15,55$ \\
\hline $\begin{array}{l}\text { Usage des œufs non } \\
\text { incubés (\%) }\end{array}$ & $\begin{array}{l}\text { Vente } \\
\text { Consommation } \\
\text { familiale } \\
\text { Don }\end{array}$ & $\begin{array}{l}5,83 \\
90,83 \\
3,34\end{array}$ & $\begin{array}{l}10 \\
88,33 \\
1,67\end{array}$ & $\begin{array}{l}22,5 \\
79,17 \\
0\end{array}$ & $\begin{array}{l}12,78 \pm 8,67 \\
86,11 \pm 6,14 \\
5,01 \pm 1,67\end{array}$ \\
\hline $\begin{array}{l}\text { Présence des casses } \\
\text { durant l'incubation (\%) }\end{array}$ & $\begin{array}{l}\text { Oui } \\
\text { Non }\end{array}$ & $\begin{array}{l}4,17 \\
95,83 \\
\end{array}$ & \begin{tabular}{|l|}
4,17 \\
95,83 \\
\end{tabular} & $\begin{array}{l}19,17 \\
80,83 \\
\end{array}$ & $\begin{array}{l}9,17 \pm 8,66 \\
90,83 \pm 8,66\end{array}$ \\
\hline \multicolumn{2}{|c|}{ Taux de casse durant l'incubation (\%) } & $0,55 \pm 3,19$ & $0,38 \pm 2,17$ & $2,96 \pm 6,59$ & $1,30 \pm 4,55$ \\
\hline Causes des casses (\%) & $\begin{array}{l}\text { Poule } \\
\text { Qualité médiocre } \\
\text { du nid } \\
\text { Ecrasement } \\
\text { Autre }\end{array}$ & $\begin{array}{l}59,95 \\
0 \\
0 \\
40,05\end{array}$ & $\begin{array}{l}79,86 \\
0 \\
40,05 \\
0 \\
\end{array}$ & $\begin{array}{l}86,96 \\
13,04 \\
0 \\
0\end{array}$ & $\begin{array}{l}75,59 \pm 14,00 \\
4,35 \pm 7,53 \\
13,35 \pm 23,12 \\
13,35 \pm 23,12\end{array}$ \\
\hline Usage œufs non éclos (\%) & $\begin{array}{l}\text { Alimentation } \\
\text { Poubelle }\end{array}$ & $\begin{array}{l}18,33 \\
92,5\end{array}$ & \begin{tabular}{|l|}
50 \\
68,33 \\
\end{tabular} & $\begin{array}{l}44,17 \\
70,83 \\
\end{array}$ & $\begin{array}{l}37,5 \pm 16,86 \\
77,22 \pm 13,29\end{array}$ \\
\hline $\begin{array}{l}\text { Taux de sevrage (\%) } \\
\text { Mortalité avant sevrage (\%) } \\
\text { Durée sevrage (mois) }\end{array}$ & & $\begin{array}{l}52,52 \pm 21,18 \\
46,64 \pm 21,06 \\
2,14 \pm 0,99 \\
\end{array}$ & $\begin{array}{l}60,39 \pm 18,93 \\
39,09 \pm 19,15 \\
3,01 \pm 1,38 \\
\end{array}$ & $\begin{array}{l}58,84 \pm 20,96 \\
41,99 \pm 21,13 \\
2,40 \pm 1,29\end{array}$ & $\begin{array}{l}57,25 \pm 20,61 \\
42,57 \pm 20,65 \\
2,52 \pm 1,28 \\
\end{array}$ \\
\hline
\end{tabular}

Contrainte liés à l'élevage de la poule locale au Sud-Kivu : Les principales contraintes identifiées pour l'élevage des poules locales sont variables selon l'âge de la poule. Parmi ces facteurs de diminution, on a principalement pour les poussins, la recrudescence des épidémies $(65,56 \pm 15,42 \%)$, les prédations $(60,56 \%)$ par les oiseaux, les chiens et parfois d'autres animaux sauvages, les intempéries et l'écrasement. Pour les poules adultes les épidémies de maladies $(82,78 \%)$ reste la principale contrainte à côté du vol $(47,5 \%)$ et la prédation $(40,56 \%)$. Les prédateurs les plus rencontrés à part l'homme $(78,89 \pm 4,27 \%)$ lors de vol restent divers animaux sauvages $(28,89 \%)$ et les chiens $(8,61 \%)$. 

développement au Sud-Kivu, Est de la République Démocratique du Congo

Tableau 4 : Principales contraintes liées à l'élevage de la poule locale au Sud-Kivu (\%)

\begin{tabular}{l|l|l|l|l|l}
\hline & \multirow{2}{*}{ Modalités } & \multicolumn{3}{l}{ Zone d'étude } & \multicolumn{2}{l}{\begin{tabular}{l} 
Maute \\
\cline { 3 - 6 }
\end{tabular}} & $\begin{array}{l}\text { Basse } \\
\text { altitude }\end{array}$ & $\begin{array}{l}\text { Moyen } \\
\text { altitude } \\
\text { altitude }\end{array}$ & Moyenne \\
\hline \multirow{4}{*}{ Contrainte aux poussins } & Maladie & 83,33 & 55,83 & 57,5 & $65,56 \pm 15,42$ \\
& Ecrasement & 5 & 14,17 & 12,5 & $10,56 \pm 4,88$ \\
& Prédation & 56,67 & 72,5 & 52,5 & $60,56 \pm 10,55$ \\
& Intempérie & 10,83 & 25,83 & 23,33 & $20 \pm 8,04$ \\
\hline \multirow{3}{*}{ Contrainte aux poules adultes } & Vol & 46,67 & 44,17 & 51,67 & $47,5 \pm 3,82$ \\
& Prédation & 37,5 & 40,83 & 43,33 & $40,56 \pm 2,92$ \\
& Maladie & 91,67 & 85 & 71,67 & $82,78 \pm 10,18$ \\
\hline \multirow{3}{*}{ Principaux prédateurs } & Homme & 80 & 74,17 & 82,5 & $78,89 \pm 4,27$ \\
& Chien & 1,67 & 13,33 & 10,83 & $8,61 \pm 6,14$ \\
& Animaux sauvages & 20,83 & 37,5 & 28,33 & $28,89 \pm 8,35$ \\
\hline
\end{tabular}

\section{DISCUSSION}

L'élevage des poules locales est principalement une affaire des hommes malgré une faible représentation de femme. Ces résultats sont similaires avec les études antérieures menées dans le même milieu (Mugumaarhahama et al., 2016). Cependant cette situation diffère de celle rapportée au Senegal et au Cameroun (Ly et al., 1999 ; Fotsa, 2008) où on signale une proportion de femmes détentrices de poules locales plus importante que celle des hommes, cela étant justifié par une pauvreté des femmes dans les milieux ruraux. Au Sud-Kivu, la poule joue un rôle important dans la scolarisation des enfants et le soutien de l'économie familiale, le fait que ces activités sont généralement sous la responsabilité de l'homme expliquerait leur proportion élevée au Sud-Kivu comme le fut observé aussi au Kenya (Ndengwa et al., 2001). Malgré son importance dans la vie des exploitants, il est constitué une activité secondaire malgré l'important gain du point de vue macroéconomique qu'il procure aux paysans, comme c'est le cas dans d'autres pays africains (Bantiéni and Modibo, 2000 ; Fotsa, 2008; Larivière, et Leroy, 2008). Dans les exploitations rurales du Sud-Kivu, la taille du cheptel varie entre 8,44 à 40,32 poules en moyenne) par exploitant. Ces effectifs sont comparables aux chiffres rapportés dans d'autres pays africains (Fotsa, 2008) où la moyenne est 10 poules par exploitant. Dans ces exploitations les coqs sont en petits nombre car ils sont de préférence réformés pour le soutien financier dans les ménages ruraux tel que observé aussi par Agbédé et al., (1993). Les poules rurales sont en majorité gardées en divagation dans les trois zones agro-écologiques et les élevages n'ont pas d'abris appropriés pour le protéger contre les intempéries, les vols et la prédation pendant les nuits ainsi pour assurer cette fonction, la majorité d'aviculteurs utilisent leurs propres maisons et lorsqu'il n'ya pas de logement, les poules sont perchées sur des arbres. Cela est une réalité dans d'autres régions rurales en Afrique (Buldgen et al., 1992). II n'existe aucun plan de rationnement pour plusieurs, ils distribuent chacun selon leur gré le matin et le soir. Cela est souvent observé durant la période de campagne agricole où les poules sont maintenues en claustration pour éviter les ravages causés aux cultures de haricot. La Newcastle disease (Pseudopeste aviaire) est la plus fréquente dans ces élevages.La pharmacopée traditionnelle est la pratique utilisée comme cheval de bataille pour le soin dans ces élevages; ceci était observé aussi au Cameroun (Buldgen et al., 1992). Une recherche beaucoup plus approfondie est nécessaire pour la valorisation de ce secteur. Les poules ainsi élevées dans ces conditions difficiles enregistrent une production de 9,67 à 16,77 œufs par cycle et une durée de sevrage de 2,52 mois contre 4 à 6 semaines observés dans d'autres milieux (Buldgen et al., 1992). Les 41,92 à 50,08 œufs obtenus par an et par poule sont comparable aux résultats trouvésen Côte d'ivoire (Kouadio et al., 2010). Le taux d'éclosion des œufs de $84,5 \%$ est toute fois supérieur à celui obtenus dans les élevages au GuinéeConakry (71\%) (Mourad et al., 1997) et comparable à celui du Cameroun (82\%) (Agbédé et al., 1995). Cependant une étude antérieure dans ce même milieu a trouvé un taux d'éclosion de $88.8 \%$ (Mugumaarahama et al., 2016). Le faible taux de sevrage des poussins au Sud-Kivu a déjà été rapporté par d'autres chercheurs pour d'autres pays (Kouadio et al., 2010). II serait lié aux épidémies, à la prédation qui 
arrivent même à décimer tout le cheptel, à un faible encadrement des exploitants et à un système d'élevage contraignant (Kouadio et al., 2010; Sarkar et Bell, 2006 ; Sonaiya,et Swan, 2004). La principale cause de

\section{CONCLUSION}

Quoique cet élevage soit une activité secondaire et selon les techniques de production traditionnelle avec des faibles performances, il reste primordiale dans l'économie des familles rurales et mérite une attention particulière visant son amélioration. Une stabulation ainsi qu'une alimentation équilibrée permettraient

\section{REMERCIEMENTS}

Nous exprimons notre profonde reconnaissance au Professeur MUSHAGALUSA NACHIGERA Gustave pour avoir apporté des remarques constructives durant la rédaction de ce travail. Notre gratitude aussi envers

\section{REFERENCES}

Agbédé, G., Nkenfou, J. Et Mpoame M., 1993. Essais préliminaires d'utilisation de Kalanchoe crenata (crussulacée) dans la prophylaxie et le traitement de la coccidiose aviaire, Tropicultura, 11(3) 107-109.

Agbédé, G., Teguia, A. et Manjeli, Y., 1995. Enquête sur l'élevage traditionnel des volailles au Cameroun, Tropicultura, 1995, 13,1, 22-24.

Bachir, P.M., Triki-Yamani, R.R., Mohamed, S.R., Stella M., Hussain, A. (2011). Evaluation of Animal Performance in Broiler Farming in the Central Region of Algeria. Bulletin UASVM, Veterinary Medicine 68(1).62-68.

Bantiéni, T. and Modibo, S., 2000, In: Proceedings of International workshop December 9-13, 1997 M'Bour, Senegal, 111-131.

Barua, A. and Yoshimura, Y., 1997. Rural poultry keeping in Bangladesh, World's Poult. Sci.J, 53, 387-394.

Bessadok, A., Khochilef, I., El Gazzah, M., 2003. Etat des ressources génétiques de la population locale du poulet en Tunisie, Tropicultura, 21, 167-172.

Buldgen, A., Detimmerman, F., Sall, B., Compere, R., 1992. Etude des paramètres démographiques et zootechniques de la poule locale du bassin arachidier sénégalais. Rév. Elev. Méd. Vét. Pays Trop., 45 (3-4) : 341-347.

DSRP (document de stratégie de la réduction de la pauvreté), 2005. Monographie de la province du Sud-Kivu: République démocratique du la diminution des effectifs reste les épidémies, la prédation et les vols comme cela a été observé aussi au Cameroun (Agbédé et al., 1993).

d'accroitre la production et réduire les risques de perte des poules. Une caractérisation phénotypique ainsi que la pharmacopée traditionnelle sont autant des questions urgentes qui nécessitent une attention particulière.

tous les fermiers de la poule locale du Sud-Kivu pour la contribution apportée en mettant à notre portée toutes les informations nécessaires lors de nos enquêtes sur terrain.

Congo, Ministère du Plan, Gombe, Kinshasa, P11

Evali D., 1996, Contribution à l'étude de l'évaluation de l'efficacité de la protection vaccinal et vérification de l'effet positif du déparasitage sur la réponse immunitaire en aviculture traditionnelle dans les régions de Kaolack et de Fatick (Sénégal), Th .Méd. Vét., Dakar, 20.

FAO, 2010. $6^{\text {th }}$ Session of the intergovernmental technical working group on animal genetic resources for food and agriculture, Rome (Italy). 92p.

Fotsa J. C., 2008. Caractérisation des populations de poules locales (Gallus Gallus) au Cameroun. Thèse, Institut national agronomique, ParisGrignon, 301p.

Gueye, E. F., 2003, Aviculture familiale et aviculture industrielle - Coopération au lieu de compétition, Bulletin RIDAF, 13 (2), 1-2.

Gueye, E. F., 2005, L'aviculture familiale ne doit plus être une " moisson cachée ». Bulletin RIDAF, $15(1), 1-2$.

INS (Institut National de la Statistique) RD Congo (2016). Annuaire statistique 2015, 560p

Khan, A.G., 2004, Réplique du poulet indigène avec son acceptabilité sur le marché est un facteur clé en aviculture familiale, Bulletin RIDAF, 14 (1): 2-11.

Kouadio, K.E., Kouao, B.J., Fantodj, A., Yapi, A., Yrebe, L., 2010. Influence du système 
d'élevage sur la mortalité des poulets locaux, CNRA, J. Appl. Biosci. 32: 2020-2026.

Larivière, J. M. et Leroy, P., 2008, Conservation et valorisation de la diversité des ressources génétiques du poulet en Europe : initiatives et perspectives ; Université de liège, Belgique.

Lariviere, J.M. and Leroy, P., 2005. Poultry biodiversity in Belgium. In: World Poultry Science Association, Proceedings of the 4th European Poultry Genetics Symposium, Dubrovnik, Croatia, 6-8 October, 22.

Legrand, D., 1988. Situation actuelle de l'aviculture sénégalaise: types et Méthodes d'élevage des poulets de chair et des pondeuses. Th. Méd. Vét., Dakar; $n^{\circ} 3$.

Loukou, N.E., Yapi-Gnaore, C.V., Toure, G., Coulibaly, Y., Rognon, X., Kayang, B., Youssao, I., Tixier-Boichard, M., N'guetta, A.S.P., 2009. Journal of Animal \& Plant Sciences, 5 (1) 425 436.

Ly, C., Savane, M., Seck, M.T., Faye, A., 1999, L'Aviculture au Sud du Sénégal. Cahiers Agricultures, $8: 123-125$.

Moula, N., Antoine-Moussiaux, N., Farnir, F., Detilleux, J., Leroy, P., 2009. Réhabilitation socioéconomique d'une poule locale en voie d'extinction : la poule Kabyle (Thayazit lekvayel), Université de Liège, Liège, Belgique.

Moula, N., 2012. Biodiversité avicole dans les pays industrialisés et en développement caractérisation et étude des performances de production de races gallines locales. Thèse, Académie universitaire Wallonie-Europe, Université de Liège ; 261.

Mourad, M., Bah, A.S., Gbanamou, G., 1997. Evaluation de la productivité et de la mortalité de la poule locale sur le plateau de Sankaran, Fanarah, (Guinée). Rév .El. Méd. Pays Trop., 50 (4), 343-349.

Munyuli, T., Mushamabanyi, B. et Balezi, N., 2002. Utilisation des blattes et des termites comme substituts potentiels de la farine de viande dans l'alimentation des poulets de chair au Sud-Kivu, République Démocratique du Congo, Tropicultura, 20 (1) 10-16.

Ndegwa, JM. , Mead, R. Norrish, P., Kimani, P.W., Wachira, AM. (2001). The Growth Performance of Indigenous Kenyan Chickens Fed Diets Containing Different Levels of Protein during Rearing. Tropical Animal Health and Production, 33; 441- 448.
Ngou Ngoupayou, J.D., 1990. Country report rural poultry production in Cameroon, In: CTA Seminar Proceedings. Smallholder Rural Poultry Production. Thessalonica, Greece, 2, 39-47.

Osei-Amponsah, R., Kayang, B.B., Naazie, A., Osei, Y.D., Youssao, A.K.I., Yapi-Gnaore, V., TixierBoichard, M., Rognon, X., 2010. Animal Science. J., 81, (3), 297-303.

Periquet, J.C., 1994, Les poules, oies et canards: races, soins, élevage, Paris: Ed. Rustica, 159 p.

Rashid, M.M., 2003. Nutritional status of scavenging chickens with special emphasis on energy and protein supplementation under rural conditions in Bangladesh. (Master of Science). Royal Veterinary and Agricultural University: Denmark, $38 \mathrm{p}$.

Sankar, K. et Bell, J.G., 2006. Potentiel du poulet indigène et son rôle dans la lutte contre la pauvreté et dans la sécurité alimentaire pour les ménages ruraux. Réseau International pour le Développement de l'Aviculture Familiale, 16 (2), 16-28.

Sonaiya, E.B. et Swan, S.E., 2004. Production en Aviculture familiale. Organisation Des Nations Unies pour l'Alimentation et l'Agriculture : Rome, $140 \mathrm{p}$.

Tchoumboue, J., Manjeli, Y., Teguia, A., Ewane, N.J., 2000, Productivité et effets comparés de trois systèmes de conduite de l'élevage sur les performances de l'aviculture villageoise dans les hautes terres de l'Ouest Cameroun, Sci. Agron. Dévelop. Prod. Anim., 6-14.

Youssao, A.K.I., Rognon, X., Yapi-Gnaore, V., Kpodekon, T.M., Idrissou, N.D., Kayang, B., Houinsou, S.A., Tixier-Boichard, M., 2009. 5th World Poultry Conference, 10 au 13 mars 2009, Taba, Egypt. 